Editorial

\title{
Special issue on Graph-Based Representations in Computer Vision
}

Graph-based representations are of pivotal importance in computer vision, pattern recognition and machine learning. They arise when the objects to be identified are decomposed into parts and relationships between them. Such representations are quite natural and find applications in low level image processing, such as segmentation or image filtering, and in high level vision tasks such as pattern matching. Graph representations also pose unique problems in machine learning, since they are non-vectorial in nature and require new methodology to be developed if they are to be learned from image data.

More generally, the continued and growing interest in graph based algorithms may be explained as follows. Current pattern recognition tasks are less and less concerned with the analysis of a single image. Rather images are grouped into sequences (video) or in massive databases disseminated over the Internet. In this setting image analysis involves the use of many image features and the analysis of their relationships. Typically this can be abstracted as a graph problem. Moreover, traditional feature-based approaches cannot satisfactorily deal with data that is naturally divided into parts (e.g., shape or text analysis) or where contextual information is essential for the classification process. For these reasons the design of efficient graph-based algorithms for pattern recognition will certainly be one of the major challenges over the next decades.

The goal of this special issue was to solicit and publish high-quality papers that provide a clear picture of the state of the art of the use of graphs as a representational tool in computer vision and pattern recognition. As a result of the call for paper we received 32 submissions, confirming that this topic stimulates lively interest in the field of Pattern Recognition and Computer Vision. Each paper was reviewed by at least two reviewers, with most being reviewed by three. This meant that we needed the assistance of some 80 reviewers which we thank for their invaluable assistance. Further, each paper was examined by the guest editors to ensure that it fell within the scope of the special issue. Based on the reviews, and giving authors the opportunity to revise their papers in the light of the reviewers comments, we selected the 14 papers that appear in this issue of Computer Vision and Image Understanding. The papers describe a diverse set of methods and applications.

The first paper presented in this special issue "Learning Graph Prototypes for Shape Recognition" by Raveaux et al. presents four new formulations of graph prototypes that can be learned using a genetic algorithm with specialized operators. The prototypes correspond to discriminative and generative models, the former yielding the best results on classification tasks.

"A Generic Framework for Median Graph Computation based on a Recursive Embedding Approach" by Ferrer et al. presents a novel and efficient approach to computing generalized median graphs by embedding into a vectorial space and recursively recovering a graph from a point in such space.

In "Models and Algorithms for computing the Common Labelling of a set of Attributed Graphs" Solé-Ribalta and Serratosa present two new approaches for the computation of a common labeling, or correspondences, from sets of graphs. The first method extends the graduated assignment algorithm, while the second adopts an iterative sub-optimal approximation.

Jain and Obermayer's paper "Graph Quantization" describes an extension of vector quantization to the graph domain. By projecting graphs into a Riemmanian orbitfold, they provide the necessary mathematical structure to allow an extension of Vector Quantization to the graph domain in a theoretically sound way.

"Graph Attribute Embedding via Riemannian Submersion Learning" by Haifeng Zhao et al. studies the problem of embedding a set of relational structures into a Riemmanian metric space. Once the map is established, a mixture of class-specific densities is defined in this space by means of charts on the embedded manifold.

"Efficient Many-to-Many Feature Matching under the 11 Norm" by Demirci et al. presents a distortion-free embedding in a geometric space under the $l_{1}$ norm. Using the flow given by the Earth Mover's Distance (EMD) algorithm in the embedding space they provide robust many-to-many vertex correspondences.

The paper "Graph-Based Quadratic Optimization: A Fast Evolutionary Approach" by Rota Bulò, Pelillo, and Bomze introduces a graph-based optimization method for the standard quadratic problem. The optimization problem is cast into a game theoretic setting where infection and immunization processes are used to yield a growth direction that has linear time complexity for each iteration.

In their paper "Polynomial Algorithms for Subisomorphism of nD Open Combinatorial Maps" Damiand et al. formalize matching problems in Combinatorial Maps as on submap isomorphism problems, and provide polynomial time algorithms for solving them.

Gonzalez Diaz et al. in the paper "Invariant Representative Cocycles of Cohomology Generators using Irregular Graph Pyramids" study topological invariants defined by cohomology over graph pyramids. The technique is applied to compute scanning and rotation invariants.

In their paper "Learning invariant structure for object identification by using graph methods" Bai, Song, and Hall, propose the use of topological structure as class invariants for classification. The authors show that structural approaches can be used to learn class identity even under widely varying appearance and even under depictive styles.

Jiang et al.'s "Graph-Based Markerless Registration of City Maps Using Geometric Hashing” robustly registers images of a small portion of a map to a much larger stored digital map. The method 
uses a geometric hashing technique of the adjacency map for city streets.

In "Bone Graphs: Medial Shape Parsing and Abstraction" Macrini et al. propose a novel shape representation based on the ligature structure of the medial axis. This provides a stable description of an object's parts.

In "2.5D Elastic Graph Matching" Zafeiriou and Petrou introduce an Elastic Graph Matching algorithm that matches range images of a face to a full 3D model for face recognition.

Finally, in their paper "Pose-invariant Face Recognition by Matching on Multi-resolution MRFs linked by Supercoupling Transform" Rahimzadeh Arashloo, Kittler, and Christmas propose an MRF-based classification approach for the recognition of faces in arbitrary pose by incorporating an image matching term within the definition of the field energy.

The variety and high quality of the submitted papers demonstrate that the topics covered by the special issue are of topical interest to the computer vision community. We hope that this collection of papers will provide both a timely and interesting sample of research in the field of graph-base representation and that will prove of lasting utility to practitioners in the field.

Edwin R. Hancock

University of York, UK

Andrea Torsello

Università Ca' Foscari Venezia, Italy

Francisco Escolano University of Alicante, Spain

Luc Brun

GREYC, CNRS UMR 6072, ENSICAEN, France 